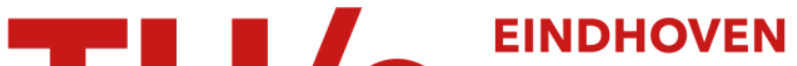

\section{Active nematic flows confined in a two dimensional channel with hybrid alignment at the walls}

\section{Citation for published version (APA):}

Rorai, C., Toschi, F., \& Pagonabarraga, I. (2021). Active nematic flows confined in a two dimensional channel with hybrid alignment at the walls: A unified picture. Physical Review Fluids, 6(11), [113302].

https://doi.org/10.1103/PhysRevFluids.6.113302

DOI:

10.1103/PhysRevFluids.6.113302

Document status and date:

Published: 30/11/2021

\section{Document Version:}

Publisher's PDF, also known as Version of Record (includes final page, issue and volume numbers)

\section{Please check the document version of this publication:}

- A submitted manuscript is the version of the article upon submission and before peer-review. There can be important differences between the submitted version and the official published version of record. People interested in the research are advised to contact the author for the final version of the publication, or visit the $\mathrm{DOI}$ to the publisher's website.

- The final author version and the galley proof are versions of the publication after peer review.

- The final published version features the final layout of the paper including the volume, issue and page numbers.

Link to publication

\section{General rights}

Copyright and moral rights for the publications made accessible in the public portal are retained by the authors and/or other copyright owners and it is a condition of accessing publications that users recognise and abide by the legal requirements associated with these rights.

- Users may download and print one copy of any publication from the public portal for the purpose of private study or research.

- You may not further distribute the material or use it for any profit-making activity or commercial gain

- You may freely distribute the URL identifying the publication in the public portal.

If the publication is distributed under the terms of Article 25fa of the Dutch Copyright Act, indicated by the "Taverne" license above, please follow below link for the End User Agreement:

www.tue.nl/taverne

Take down policy

If you believe that this document breaches copyright please contact us at:

openaccess@tue.nl

providing details and we will investigate your claim. 


\title{
Active nematic flows confined in a two-dimensional channel with hybrid alignment at the walls: A unified picture
}

\author{
C. Rorai, ${ }^{1}$ F. Toschi $\odot,^{2}$ and I. Pagonabarraga ${ }^{1,3,4}$ \\ ${ }^{1}$ CECAM, Centre Européen de Calcul Atomique et Moléculaire, \\ École Polytechnique Fédérale de Lausanne (EPFL), Batochime, \\ Avenue Forel 2, 1015 Lausanne, Switzerland \\ ${ }^{2}$ Department of Applied Physics, Fluid Dynamics and Heat Transfer, \\ Technical University of Eindhoven (TU/e), 5612 AZ Eindhoven, the Netherlands \\ ${ }^{3}$ Departament de Física de la Matèria Condensada, Universitat de Barcelona, \\ C. Martí Franquès 1, 08028 Barcelona, Spain \\ ${ }^{4}$ University of Barcelona Institute of Complex Systems (UBICS), \\ Universitat de Barcelona, 08028 Barcelona, Spain
}

(Received 9 August 2021; accepted 5 November 2021; published 30 November 2021)

Active nematic fluids confined in narrow channels are known to generate spontaneous flows when the activity is sufficiently intense. Recently, it was demonstrated [R. Green, J. Toner, and V. Vitelli, Phys. Rev. Fluids 2, 104201 (2017)] that if the molecular anchoring at the channel walls is conflicting, i.e., perpendicular on one plate and parallel on the other, flows are initiated even in the zero activity limit. An analytical laminar velocity profile for this specific configuration was derived within a simplified nematohydrodynamic model in which the nematic order parameter is a fixed-magnitude unit vector $\mathbf{n}$. The solution holds in a regime where the flow does not perturb the nematic order imposed by the walls. In this study, we explore systematically active flows in this confined geometry with a more general theoretical model that uses a second-rank tensor order parameter $\mathbf{Q}$ to express both the magnitude and orientation of the nematic phase. The $\mathbf{Q}$-model allows for the presence of defects and biaxial, in addition to uniaxial, molecular arrangements. Our aim is to provide a unified picture, beyond the limiting regime explored previously, to serve as a guide for potential microfluidic applications that exploit the coupling between the orientational order of the molecules and the velocity field to finely control the flow and overcome the intrinsic difficulties of directing and pumping fluids at the microscale. We reveal how the nematic-flow coupling is not only dependent on geometrical constraints, but is also highly sensitive to material and flow parameters. We specifically stress the key role played by the activity and the flow aligning parameter, and we show that solutions mostly depend on two dimensionless parameters. We find that for large values of the activity parameter, the flow is suppressed for contractile particles while it is either sustained or suppressed for extensile particles depending on whether they tend to align or tumble when subject to shear. We explain these distinct behaviors by an argument based on the results of the stability analysis applied to two simpler configurations: active flows confined between parallel plates with either orthogonal or perpendicular alignment at both walls. We show that the analytical laminar solution derived for the $\mathbf{n}$ model in the low activity limit is found also in the $\mathbf{Q}$ model, both analytically and numerically. This result is valid for both contractile and extensile particles and for a flow-tumbling as well as aligning nematics. We remark that this velocity profile can be derived for generic boundary conditions. To stress the more general nature of the $\mathbf{Q}$ model, we conclude by providing a numerical example 
of a biaxial three-dimensional thresholdless active flow for which we show that biaxiality is especially relevant for a weakly first-order isotropic-nematic phase transition.

DOI: 10.1103/PhysRevFluids.6.113302

\section{INTRODUCTION}

Active fluids constitute a special class of complex fluids characterized by the presence of an active phase that consists of, for example, microorganisms, actomyosin networks or self-propelled colloids [1]. In these liquids the active component is able to sustain flows by continuously injecting energy at the scale of its single constituents.

Numerous earlier studies have shown a compelling qualitative and quantitative correspondence between behaviors predicted by continuum active nematohydrodynamic models [2,3] and phenomena observed in a variety of active fluid systems [4-7]. Supported by this evidence, we focus on studying the behavior of active nematic liquid crystals, a class of apolar materials that display orientational order and whose particles self-propel. In the mathematical model the motility is accounted for by an active force term derived by considering that active particles can be approximated to leading order as force dipoles [3]. In these systems the transition between a passive state, in which activity is macroscopically incoherent, and an active state, characterized by a spontaneous active flow, is generally observed above a certain activity threshold [8-12]. However, there exists a family of flows that violates this rule by developing steady state velocity fields even for vanishingly small activity.

The existence of thresholdless active flows was first reported numerically [13] and later formalized theoretically [14] by identifying the asymptotic parameter regime required for their onset and the topological constraints, boundary conditions and external forcing that allow for them. A nonuniform, minimum energy nematic profile, geometrically constrained and leading to a nonvanishing curl active force constitutes the key ingredient for such a class of fluids [14]. A realization of this situation is achieved with an active nematic liquid confined between parallel plates with hybrid anchoring at the walls: parallel on one plate, perpendicular on the other; this is one of the examples presented in [14] and studied in [13] and this is the setting our study focuses on. Configurations with the same anchoring at both walls, e.g., parallel anchoring or perpendicular anchoring, lead to uniform ground states which can support a coherent unidirectional active flow only above well defined thresholds for the activity parameter as derived through the linear stability analysis $[8,10]$.

The motivation for studying active flows confined in a slab geometry with hybrid anchoring at the walls is of both applied and theoretical nature. On one hand this configuration is relevant to microfluidic applications, on the other, the results reported in the literature [13,14] differ and call for a more comprehensive unified picture.

In nematic liquid crystals the coupling between the orientational order of the molecules and the flow is controlled by several material and flow parameters and the nematic configuration is highly sensitive to geometrical constraints. The emerging complex dynamics of these active liquids is of great promise for microfluidic applications since it provides a means to control and finely tune the flow overcoming the intrinsic difficulties of directing and pumping isotropic fluids at the microscale [15-17]. Devices that direct and sort nano and micro-particles have already been presented in the literature: some exploit the anisotropic nature of the fluid to control the flow resistance and streamlines through the application of external electrical fields [18], some use defect lines as rails to transport colloids $[19,20]$ in what is referred to generically as topological microfluidics. Recently, it has been conceptualized how active liquid crystal can be exploited to design autonomous microfluidic devices [21]. Numerical studies have also appeared to shed light on the active flow dynamics and transition from coherent to turbulent state in two or three dimensional microchannels [22,23].

The numerical [13] and theoretical [14] studies, we will mainly refer to, are performed in two different frameworks: in [14] the hydrodynamic active nematic equations are expressed in terms of the director field $\mathbf{n}$, which represents the average long axis orientation for rodlike molecules, while 
in [13] the nematic is described by a more general tensor order parameter $\mathbf{Q}$ that expresses both the magnitude, $q_{0}$, and orientation, $\mathbf{n}$, of the nematic phase. The tensor order parameter formulation naturally embodies defects and allows for biaxial states [24] in three-dimensions.

The active nematic equations expressed in $\mathbf{Q}$ and $\mathbf{n}$ coincide for a uniaxial nematics with uniform $q_{0}$ up to second order terms in $\mathbf{n}$ (see Appendix $\mathrm{C}$ in [13]), yet the, respectively, numerical and analytical results reported in [13] and [14] for a low activity laminar flow confined between parallel plates with hybrid alignment at the walls differ. The aim of this work is therefore twofold: (i) provide a unifying picture for this class of active flows that generalizes the regimes explored previously and bridges the analytical results derived in [14] with the numerical ones in [13] and (ii) take advantage of the more general nature of the $\mathbf{Q}$ formulation and explore the existence of biaxial thresholdless active flows.

In this paper, we first present the mathematical and numerical model we use (Sec. II A) and list a complete set of dimensionless numbers that characterize the dynamics and associated relevant regimes (Sec. II B). We then show, in part III A, that the laminar flow solution derived for the $\mathbf{n}$ model in the low activity limit [14] satisfies also the $\mathbf{Q}$ model for an appropriate choice of the free-energy parameters. More specifically, in Sec. III A 1, we find that the analytical solution found by Green et al. [14] can be recovered with the two-dimensional $\mathbf{Q}$ model and we remark that this solution can be generalized to any choice of the anchoring angle. Although no analytical expression is found for a thresholdless active flow given a three-dimensional $\mathbf{Q}$ tensor, in Sec. III A 2 we show that the two-dimensional solution is a very good approximation for the three-dimensional solution found numerically. Details are also given on the parameter values required to observe such flows numerically. In Sec. III B we compute numerically steady state solutions in a wide portion of parameter space and show that they mainly depend on two dimensionless groups: the flow aligning parameter and a number that quantifies the distance from the low activity limit and a regime where the flow does not perturb the nematic order imposed by the walls. We find that for large values of the activity the flow is suppressed for contractile particles while is either sustained or suppressed for extensile particles depending on whether they tend to align or tumble when subject to shear. We explain these distinct behaviors in Sec. III C by an argument based on the results of the stability analysis applied to two simpler configurations: active flows confined between parallel plates with either orthogonal or perpendicular alignment at both walls. We find that the zero-flow solution selected dynamically by the system for a contractile nematic corresponds to a free energy stationary point that is not admitted in the $\mathbf{n}$ model. In Sec. III D we compare this zero-curl stationary point with the thresholdless flow solution. Finally, to stress the more general nature of the $\mathbf{Q}$ model, we provide a numerical example of a biaxial three-dimensional thresholdless active flow and we show that biaxiality is especially relevant for a weakly first-order isotropic-nematic phase transition, Sec. III E. We conclude by summarizing our findings in Sec. IV.

\section{MATHEMATICAL AND NUMERICAL MODEL}

\section{A. The $\mathbf{Q}$ hydrodynamical model for active nematics}

In the tensor order parameter model, the nematic is described by a second-order tensor $Q_{i j}$ that expresses both the magnitude $q_{0}$, and orientation $\mathbf{n}$, of the nematic phase. The tensor orderparameter formulation naturally embodies defects and allows for biaxial states [24], in fact $Q_{i j}$ can be generically expressed as

$$
Q_{i j}=q_{0} n_{i} n_{j}-q_{1} m_{i} m_{j}-\left(q_{0}+q_{1}\right) \frac{\delta_{i j}}{d},
$$

where $\mathbf{n}$ and $\mathbf{m}$ are perpendicular directors of unit length that represent the axes of reflection symmetry of a biaxial nematic, $q_{0}$ and $q_{1}$ are the associated magnitudes, and $d$ is the spatial dimension of the problem. Biaxiality is possible only in three dimensions (3D). For a uniaxial nematic, $q_{1}=0$, and $\mathbf{n}$ is an axis of rotational symmetry; in this case, the order parameter reduces to $Q_{i j}=q_{0}\left(n_{i} n_{j}-\delta_{i j} / d\right)$. 
In the Q-model it is customary to adopt the Landau-de Gennes free energy, which consists of a distortion term multiplied by the elastic constant $K$ and bulk terms with constants $A, B$, and $C$ that represent the thermotropic part of the free energy [2,3,24],

$$
\mathcal{F}=\int d^{3} r\left[\frac{K}{2}\left(\partial_{k} Q_{i j}\right)^{2}+\frac{A}{2} Q_{i j} Q_{j i}+\frac{B}{3} Q_{i j} Q_{j k} Q_{k i}+\frac{C}{4}\left(Q_{i j} Q_{j i}\right)^{2}\right] .
$$

The molecular field tensor is then defined as

$$
\begin{aligned}
\mathcal{H}_{i j} & =-\frac{\delta \mathcal{F}}{\delta Q_{i j}}+\frac{\delta_{i j}}{d} \operatorname{Tr} \frac{\delta \mathcal{F}}{\delta Q_{k l}} \\
& =K \nabla^{2} Q_{i j}-A Q_{i j}-B Q_{i k} Q_{k j}-C\left(Q_{l k} Q_{k l}\right) Q_{i j}+B \frac{\delta_{i j}}{d}\left(Q_{l k} Q_{k l}\right) .
\end{aligned}
$$

For a uniaxial nematic and $d=2$ and 3, Eq. (3) simplifies, respectively, into

$$
\begin{gathered}
\mathcal{H}_{i j}=K \nabla^{2} Q_{i j}-\left(A+\frac{C}{2} q_{0}^{2}\right) Q_{i j} \quad(d=2), \\
\mathcal{H}_{i j}=K \nabla^{2} Q_{i j}-\left(A+\frac{B}{3} q_{0}+\frac{2}{3} C q_{0}^{2}\right) Q_{i j} \quad(d=3) .
\end{gathered}
$$

The active nematic equations with $\Gamma$ as the rotational diffusivity and $\rho$ as the fluid density read

$$
\begin{gathered}
\partial_{i} u_{i}=0, \\
\left(\partial_{t}+u_{k} \partial_{k}\right) u_{i}=\frac{1}{\rho} \partial_{j} \Pi_{i j}, \\
\left(\partial_{t}+u_{k} \partial_{k}\right) Q_{i j}-S_{i j}=\Gamma \mathcal{H}_{i j},
\end{gathered}
$$

where Eq. (6) imposes the incompressibility condition on the velocity field $u_{i}$, Eq. (7) is the NavierStokes equation with pressure term $\Pi_{i j}$, and Eq. (8) describes the evolution of the nematic tensor with $S_{i j}$ as the corotation term. The pressure term is

$$
\begin{aligned}
\Pi_{i j}= & -P \delta_{i j}+2 \eta E_{i j}+2 \xi\left(Q_{i j}+\delta_{i j} / d\right)\left(Q_{k l} \mathcal{H}_{l k}\right)-\xi \mathcal{H}_{i k}\left(Q_{k j}+\delta_{k j} / d\right)-\xi\left(Q_{i k}+\delta_{i k} / d\right) \mathcal{H}_{k j} \\
& -\partial_{i} Q_{k l}\left(\delta \mathcal{F} / \delta \partial_{j} Q_{l k}\right)+Q_{i k} \mathcal{H}_{k j}-\mathcal{H}_{i k} Q_{k j}-\alpha Q_{i j},
\end{aligned}
$$

where $\alpha$ is the activity parameter. The active liquid crystal is contractile for $\alpha$ negative, and extensile otherwise. Large values of the activity parameter are expected to destabilize the nematics by triggering instabilities, eventually leading to a chaotic behavior. The corotation term is given by

$$
S_{i j}=\left(\xi E_{i k}+\Omega_{i k}\right)\left(Q_{k j}+\delta_{k j} / d\right)+\left(Q_{i k}+\delta_{i k} / d\right)\left(\xi E_{k j}-\Omega_{k j}\right)-2 \xi\left(Q_{i j}+\delta_{i j} / d\right)\left(Q_{k l} \partial_{k} u_{l}\right),
$$

where $E_{i k}$ and $\Omega_{i k}$ are, respectively, the symmetric and antisymmetric part of the velocity gradient tensor, that is, the strain rate tensor and the vorticity tensor, while the parameter $\xi$ is the flow-aligning parameter. The corotation term expresses the response of the nematic field to the extensional and rotational part of the velocity gradients, a low value of the flow-aligning parameter induces tumbling of the particles, while larger values correspond to a flow-aligning tendency. The range of $\xi$ values that correspond to a flow-tumbling and flow-aligning behavior can be found in analogy with the $\mathbf{n}$ model: when $\lambda=\xi \frac{2+q_{0} d-2 q_{0}}{q_{0} d}$ is larger than unity, particles are in the flow-aligning regime. In the case of a biaxial nematics, the flow-tumbling and flow-aligning distinction will still hold true, but the additional $q_{1}$ parameter expressing the magnitude of biaxiality will enter into the expression for $\lambda: \lambda=\xi\left(2+q_{0} d-2 q_{0}-2 q_{1}\right) /\left(q_{0} d\right)$.

The active nematohydrodynamic equations (6)-(8) are solved numerically using a hybrid lattice Boltzmann (LB) finite-difference method [25]. More precisely, the nematic pressure term and the equation for the evolution of the $Q_{i j}$ tensor are integrated through a second-order finite-difference 

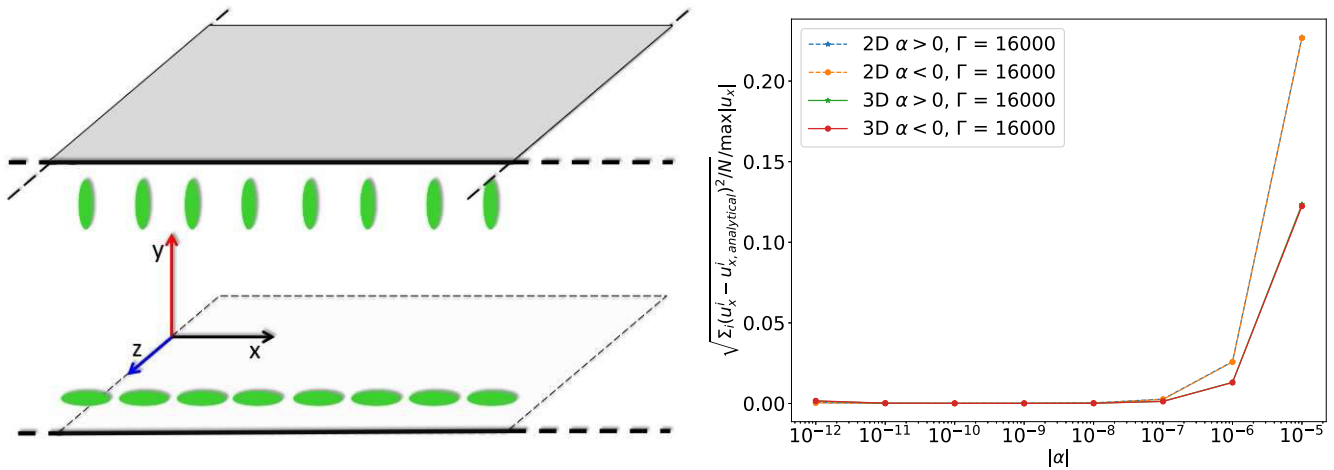

FIG. 1. Left: schematic representation of a channel with hybrid alignment at the walls. The channel walls located at $y=0$ and $y=L$ extend to infinity in the $x$ and $z$ directions. The anchoring of the active nematic liquid crystals is parallel to the $y=0$ wall (homogeneous anchoring) and normal to the $y=L$ wall (homeotropic anchoring). The numerical integration is performed in 1D. Right: Normalized root-mean-square error measuring the deviation of the numerical velocity profile from the analytical expression Eq. (12) as a function of the magnitude of the activity parameter. In the formula reported on the $y$-axes, $N$ is the number of grid-points. The numerical solution is obtained by integrating the full active nematohydrodynamic equations with either a two-dimensional or a three-dimensional tensor order parameter $Q_{i j}$. The parameters of the simulations are $v=0.33, \rho=2, L=256, t=500000, \xi=0.7, \Gamma=16000$, and $K=5 \times 10^{-6}$. For the two-dimensional case, $q_{0}=0.9998, A=-2.5 \times 10^{-6}, B=0$, and $C=5 \times 10^{-6}$; for the three-dimensional case, $q_{0}=0.5, A=0$, and $B=-C=-3 \times 10^{-5}$. These parameter values correspond to $10^{-2}<\left|\Pi_{1}\right|<10^{5}$, $\Pi_{2} \approx 6.8 \times 10^{-3}, \Pi_{3}=10560$.

scheme. The time integration of $Q_{i j}$ is performed by means of an explicit second-order AdamsBashforth time-stepping scheme. The contribution of the active and passive nematic pressure terms is added to the Navier-Stokes equation as an external forcing. The Navier-Stokes equations are then integrated through the lattice Boltzmann method [26]. The LB method makes the code ideally suited for parallel computing; the code is parallelized on CPUs with an MPI distributed parallelism.

For this study, the equations are integrated in a channel that extends from $y=0$ to $y=L$ with no-slip boundary conditions and hybrid anchoring at the walls. Specifically, for most of our calculations, the nematic order parameter is aligned parallel to the wall at $y=0$ and perpendicular to it at $y=L$, that is, for $\theta=\arctan \left(n_{y} / n_{x}\right)$ with $n_{x}$ and $n_{y}$ the $x$ - and $y$-components of the director field $\mathbf{n}$, one has $\theta(y=0)=0$ and $\theta(y=L)=\pi / 2$. Different anchoring angles have been considered in Sec. III D. See Fig. 1 (left) for a schematic representation of the geometrical configuration. We carry out the numerical integration on 1D domains. This implies that only the $x$-component of the velocity is nonzero and only the $y$-derivatives of the velocity and order tensor fields are nonzero, hence instabilities can only manifest and grow in the $y$-direction. The order parameter $Q_{i j}$ is allowed to have nonzero components on either a $2 \mathrm{D}$ plane or in the $3 \mathrm{D}$ space, that is, $Q_{i j}$ can be either two-dimensional $(d=2)$ or three-dimensional $(d=3)$. The analytical solutions in Sec. III A 1 are derived for a two-dimensional $Q_{i j}$, the numerical results reported in Sec. III A 2 are obtained for both a three-dimensional and two-dimensional $Q_{i j}$, while the numerical results shown in Secs. III B and III D are for a three-dimensional $Q_{i j}$.

\section{B. Dimensionless parameters}

Several dimensional parameters appear in Eqs. (7) and (8): $\eta, \alpha, \rho, \Gamma, K, A, B, C$. Three characteristic length scales can be identified in this model: (i) a length scale representing the core size of topological defects, $l_{c}$, (ii) an active length marking the scale at which active energy is injected into the system $[27,28], l_{a}$, and (iii) a geometrical length scale, $L$, representing the width of 
the channel. The scale of the defect core, $l_{c}$, is estimated through a Taylor series expansion around the minimum of the free energy Eq. (2). For a three-dimensional nematic tensor, this yields

$$
l_{c}=\sqrt{\frac{K}{A / 3+2 B q_{0, \mathrm{eq}} / 9+2 C q_{0, \mathrm{eq}}^{2} / 3}},
$$

where $q_{0, \text { eq }}$ is the equilibrium value of the magnitude of the nematic tensor for a uniform and undistorted nematic. The active length scale is estimated balancing the active and passive nematic terms $l_{a}=\sqrt{K /|\alpha|}$.

These three characteristic length scales combined with the characteristic velocity scale of the flow, $v_{0}$, and the dimensional parameters that do not appear in the definition of $l_{c}$ and $l_{a}$ provide the following complete set of dimensionless parameters:

(i) The balance between the inertia and viscous terms in Eq. (7) gives the Reynolds number $\operatorname{Re}=\rho v_{0} L / \eta$, note, however, that we are concerned with steady state solutions and effectively one-dimensional profiles for which the material derivatives in both Eq. (7) and (8) are zero. The Reynolds number is therefore always zero and not relevant to the problem under consideration.

(ii) A balance between the viscous terms and the passive nematic terms in Eq. (7) yields the Ericksen number $\mathrm{Er}=\eta v_{0} L / K$.

(iii) A balance between the active terms and the passive nematic terms gives the ratio between the active length scale and the system characteristic length scale: $\Pi_{1}=\alpha L^{2} / K=\operatorname{sign}(\alpha) L^{2} / l_{a}^{2}$.

(iv) The ratio between the characteristic length of the defect core and the channel length scale provides $\Pi_{2}=l_{c} / L$.

(v) Finally, the dimensionless number used to identify the frozen director limit (FDL) discussed in the following sections is $\Pi_{3}=\Gamma \eta$.

A sixth dimensionless parameter that appears in the model in dimensionless form is the flow aligning parameter $\xi$. Numerically, it is necessary to resolve all the relevant length scales, particularly the defect core, $l_{c}$, and the active length, $l_{a}$. The time scale $\tau=L^{2} / K \Gamma$ provides a useful reference on the relaxation time scale and the duration of the initial transient that precedes convergence to a steady-state solution.

Out of the six dimensionless parameters, we expect our system to be independent of $\mathrm{Re}$, as explained above, $\Pi_{2}$, since we select system sizes much larger than the characteristic defect core $\left(\Pi_{2} \ll 1\right)$, and Er since in the absence of an external forcing the characteristic velocity $v_{0}$ depends on the other model parameters. The Ericksen number will coincide with $\Pi_{1}$ when the viscous and active forces balance $\left(v_{0} \propto \alpha L / \eta\right)$, with $\Pi_{3}$ when the molecular field term and the corotation term balance in Eq. (8) $\left(v_{0} \propto \Gamma K / L\right)$, and it will be a function of $\Pi_{1}$ and $\Pi_{3}$ in all the other cases. In conclusion, we expect our problem to depend on three independent parameters: $\Pi_{1}, \Pi_{3}$, and $\xi$.

\section{RESULTS}

\section{A. Thresholdless active flow in a two-dimensional channel with mixed boundary conditions}

\section{Analytical solutions in the $\boldsymbol{n}$ and $\mathrm{Q}$ model}

As Green et al. [14] noted, in steady state and in the absence of fluid flow the equation for the evolution of the director field in the $\mathbf{n}$-model simply reduces to the Euler-Lagrange equation for minimizing the free energy with constraint $|\mathbf{n}|=1: \frac{\delta F}{\delta n_{i}}-\left(\frac{\delta F}{\delta n_{j}} n_{j}\right) n_{i}=0$, where $F$ is the Frank free energy. If the director field is in the ground state, it is shown that the velocity field is zero only if the pressure gradient balances the active force term $f_{a, i}=\partial_{j}\left(n_{j} n_{i}\right)$ exactly [14]. Hence, a sufficient condition for the onset of thresholdless active flows is that the active force has a nonvanishing curl [14]. Under this condition and in the regime where the nematic is not distorted by the flow, referred to as the FDL $\left(\Pi_{3} \gg 1\right)$, analytic expressions for the flow field can be derived. Green et al. [14] provide some solutions for various geometrical configurations, among them a two-dimensional channel flow with hybrid alignment at the walls as shown in Fig. 1 (left). 
In a $2 \mathrm{D}$ channel with walls at $y=0$ and $y=L$ and mixed boundary conditions $-n_{x}(x, 0)=1$, $n_{y}(x, 0)=0, n_{x}(x, L)=0, n_{y}(x, L)=1$ - the equilibrium nematic profile

$$
n_{x}=\cos \left(\frac{\pi y}{2 L}\right), \quad n_{y}=\sin \left(\frac{\pi y}{2 L}\right)
$$

induces an active force with nonvanishing curl [14]. In the zero activity limit and Stokes flow regime, the velocity field can be computed analytically (see Appendix G in [14]) leading to

$$
u_{x}=-\frac{\alpha L}{2 \pi \eta}\left(\cos \frac{\pi y}{L}+2 \frac{y}{L}-1\right) .
$$

Finding a solution analogous to (11) and (12) in the $\mathbf{Q}$-model requires solving $\mathcal{H}_{i j}=0$ in two dimensions with mixed boundary conditions: $n_{x}(x, 0)=1, n_{y}(x, 0)=0, n_{x}(x, L)=0, n_{y}(x, L)=1$ and the assumption of uniform $q_{0}$. Given that $Q_{i j}$ is a function of $y$ only and $|\mathbf{n}|$ is unitary, we have

$$
\frac{Q_{x x}}{q_{0}}=\frac{1}{2}-n_{y}^{2}, \quad \frac{Q_{x y}}{q_{0}}=\frac{Q_{y x}}{q_{0}}=n_{y} \sqrt{1-n_{y}^{2}}, \quad \frac{Q_{y y}}{q_{0}}=n_{y}^{2}-\frac{1}{2} .
$$

Since $q_{0}$ is uniform, Eq. (4) can be rewritten as $\mathcal{H}_{i j}=K \nabla^{2} Q_{i j}-a Q_{i j}$, where $a$ is a constant, and the stationary point condition $\mathcal{H}_{i j}=0$ corresponds to the system of ODEs,

$$
\begin{gathered}
-2\left(n_{y}^{\prime 2}+n_{y} n_{y}^{\prime \prime}\right)=\bar{a}\left(\frac{1}{2}-n_{y}^{2}\right), \\
{\left[\frac{-3 n_{y} n_{y}^{2}+2 n_{y}^{3} n_{y}^{\prime 2}+n_{y}^{\prime \prime}-3 n_{y}^{2} n_{y}^{\prime \prime}+2 n_{y}^{4} n_{y}^{\prime \prime}}{\left(1-n_{y}^{2}\right)^{3 / 2}}\right]=\bar{a} n_{y} \sqrt{1-n_{y}^{2}},} \\
2\left(n_{y}^{\prime 2}+n_{y} n_{y}^{\prime \prime}\right)=\bar{a}\left(n_{y}^{2}-\frac{1}{2}\right),
\end{gathered}
$$

for, respectively, the $x x, x y$, and $y y$ components of the molecular field. Here $n_{y}^{\prime}$ and $n_{y}^{\prime \prime}$ are, respectively, the first and second total derivative of $n_{y}$, while $\bar{a}=a / K$. Note that the first and third equations coincide. If we replace $n_{y}^{\prime \prime}=-n_{y}^{\prime 2} / n_{y}+\bar{a}\left(n_{y}^{2}-0.5\right) /\left(2 n_{y}\right)$ obtained from Eq. (14) into Eq. (15), we get $n_{y}^{\prime 2}=\bar{a}\left(n_{y}^{2}-1\right) / 4$, which, solved with the mixed boundary conditions, gives

$$
n_{y}(y)=-\frac{1}{2} i e^{-i \frac{\pi y}{2 L}}\left(1-e^{i \frac{\pi y}{L}}\right)=\sin \left(\frac{\pi y}{2 L}\right) .
$$

From Eq. (17) we have that $a=-K \pi^{2} / L^{2}$, hence for small $K$ and large $L$ (e.g., the values we have chosen for our numerical calculations: $K=5 \times 10^{-6}$ and $100 \leqslant L \leqslant 256$ ) one has $a \ll 1$. This shows that the ground-state configuration found in [14] [Eq. (11)] for mixed boundary conditions and the active nematic equations expressed in terms of the director field $\mathbf{n}$ satisfies also the active nematic equations formulated in terms of the tensor order parameter provided that $a$ is nonzero and as given above. The value of $a$ prescribes the values for the constants $A, C$, and $q_{0}$ according to expression (4).

To find the velocity profile that corresponds to the nematic profile (17), we substitute it into expression (9) and solve Eq. (7). We then have

$$
\Pi_{i j}=-P \delta_{i j}+2 \eta E_{i j}-K\left(\partial_{i} Q_{k l} \partial_{j} Q_{l k}\right)-\alpha Q_{i j},
$$

where the third term in $\Pi_{i j}$ is nonzero only for $i=j=y$, it is constant, and hence it does not contribute to Eq. (7), which, as in [14], reduces to

$$
\begin{gathered}
\eta u_{x}^{\prime \prime}-\alpha q_{0}\left(n_{y} n_{x}^{\prime}+n_{x} n_{y}^{\prime}\right)=0, \\
-P^{\prime}-\alpha q_{0}\left(n_{y}^{2}\right)^{\prime}=0,
\end{gathered}
$$

which, once solved with no-slip boundary conditions gives the same solution as in [14], here Eq. (12), except for an extra multiplicative factor $q_{0}$. 
Note that Eqs. (17) and (12) are just a special case of a broader family of solutions with anchoring conditions $\theta(y=0)=\theta_{0}$ and $\theta(y=L)=\theta_{L}$. By defining $\Delta \theta=\theta_{L}-\theta_{0}$ we have that the general solution is

$$
\begin{gathered}
n_{x}=\cos \left(\frac{\Delta \theta y}{L}+\theta_{0}\right), \quad n_{y}=\sin \left(\frac{\Delta \theta y}{L}+\theta_{0}\right), \\
u_{x}=-\frac{\alpha L q_{0}}{4 \eta \Delta \theta}\left\{\cos \left(\frac{2 \Delta \theta y}{L}+2 \theta_{0}\right)-\frac{y}{L}\left[\cos \left(2 \Delta \theta+2 \theta_{0}\right)-\cos \left(2 \theta_{0}\right)\right]-\cos \left(2 \theta_{0}\right)\right\} .
\end{gathered}
$$

The case $\Delta \theta=0$ corresponds to the degenerate case with uniform $n_{x}$ and $n_{y}$ and zero velocity. Consider also that for a 1D geometry, the zero-curl condition for the active force is satisfied whenever the off-diagonal terms of $Q_{i j}$ are zero.

The analytical solutions (11) and (12) derived for a $2 \mathrm{D} Q_{i j}$ in a 1D-geometry cannot be easily extended to the case of a 3D $Q_{i j}$. In fact, in 1D only the trivial $q_{0}=0$ solution satisfies the system $\mathcal{H}_{i j}=0$, for $\mathcal{H}_{i j}$ as in Eq. (5), mixed boundary conditions, and the simplifying assumptions of a uniaxial nematic, uniform $q_{0}$, and constant $n_{z}$. Similarly, no analytical solutions were found for the less restrictive conditions of a uniaxial nematic and (i) uniform $q_{0}$ and variable director field $n_{z}(y)$ or (ii) constant $n_{z}$ and variable $q_{0}(y)$. The stationary point solution for $\mathcal{H}_{i j}=0$ with a 3D $Q_{i j}$ can be found numerically and will simultaneously involve a nonhomogeneous $q_{0}$, a variable director field, and biaxiality. This is shown in the next section, where we also stress that for our choice of parameters the deviations from uniform $q_{0}$ and uniaxiality are small.

\section{Numerical analysis of the thresholdless active flow}

Our first aim is to verify solution (12) numerically for a 2D and a 3D Q-tensor. This velocity profile is found in the limit of small activity, $\left|\Pi_{1}\right| \ll 1$, and a "frozen" nematic, $2 q_{0} / \Gamma \ll \eta$, or, for $q_{0} \approx$ const and of order $1, \Pi_{3} \gg 1$. Reproducing (12) numerically requires a careful selection of the model parameters because deviations from its perfectly symmetric shape are significant even for small values of the coupled passive nematic terms, expression (9), and corotation terms, Eq. (10). The following considerations guided us in identifying the right parameter range to replicate (12): a stable numerical solution of the diffusion terms $\left(\partial_{t} Q_{i j}=\Gamma K \nabla^{2} Q_{i j}\right)$ in Eq. (8) for a central difference second-order Adams-Bashforth time-stepping scheme requires $\Gamma K<2 / 21$, hence the large values of $\Gamma$ called for by the FDL require correspondingly small values of $K$ and force even smaller $\alpha$ to satisfy the small activity limit.

Figure 1 (right) shows the normalized root-mean-square (RMS) deviation of the numerical results from the analytical solution as a function of the magnitude of the activity parameter $|\alpha|$. In this plot, $\Pi_{3}=10560$, while $10^{-2}<\left|\Pi_{1}\right|<10^{5}$. The numerical solution is in excellent agreement with the analytical one in the small activity limit, and it deviates from it as $|\alpha|$ increases. As expected, the deviation from solution (12) is continuous with the model parameters. In quantitative terms, we find that the RMS error is below $0.26 \%$ for $|\alpha| \leqslant 10^{-7}$ (or $\left|\Pi_{1}\right|=10^{3}$ ), suggesting that in reality the condition for small activity, $\Pi_{1} \ll 1$, holds for a wider range than predicted. We also find that for a 3D order parameter, the deviation of the minimum-energy solution from Eq. (11) is small and involves a variation of $q_{0}$ in proximity to the walls as well as a small degree of biaxiality far from the boundaries. These features have been verified numerically by letting

$$
\partial_{t} Q_{i j}=\Gamma \mathcal{H}_{i j}
$$

relax to equilibrium for a 3D Q. For $|\alpha|=10^{-12}$ the 3D n-profile shows a deviation of $\approx 0.015 \%$ from the analytical profile (17), while the variation of $q_{0}$, as well as the degree of biaxiality estimated as the difference between the two lowest eigenvalues, are approximately $\approx 0.01 \%$. Therefore, we conclude that the 2D $\mathbf{Q}$ solution is a very good approximation for the $3 \mathrm{D} \mathbf{Q}$ case.

In closing, retrieving the analytical solution (12) numerically served the double purpose of testing the code and proving that the parameter regimes where the solution exists can be accessed and explored numerically. 


\section{B. Transition from symmetric to asymmetric velocity profiles}

The velocity profile in the FDL is antisymmetric with respect to the midpoint of the channel. This symmetry is readily broken by increasing $\alpha$ (or in dimensionless terms $\left|\Pi_{1}\right|$ ) and/or decreasing $\Gamma$ (or $\Pi_{3}$ ) as a consequence of the fact that the passive nematic terms in expression (9) as well as term (10) start playing a role. The interplay between these terms and the active term also breaks the positive/negative $\alpha$-symmetry embodied in solution (12): in general, the behavior for negative $\alpha$ differs from that of positive $\alpha$. An example is provided by the low-activity asymmetric velocity profiles reported in Figs. 13-15 in [13].

In this section, we explore how the velocity and nematic profiles evolve in parameter space moving away from the FDL regime. The aim is to expand on previous studies and provide a unified picture that includes both the laminar profile derived in [14], or more precisely, its numerical analog for a 3D Q tensor (see Sec. III A 2), and the numerical results reported in [13]. We quantify the deviations from the theoretical prediction, Eq. (12), through the ratio between the maximum magnitude of the velocity and the maximum of the analytical profile: when the solution deviates from (12), this quantity departs from unity.

In Figs. 2(a) and 2(b), we show the behavior of the rescaled maximum magnitude of the velocity in a logarithmic scale for negative and positive values of the activity parameter as a function of $\Pi_{3}$ and $\left|\Pi_{1}\right|$ with $\Pi_{3}$ ranging over almost four orders of magnitude: $6.67<\Pi_{3}<2 \times 10^{4}$, and $\left|\Pi_{1}\right|$ spanning over six orders of magnitude: $20<\left|\Pi_{1}\right|<2 \times 10^{7}$. As a comparison, in [13] $125<$ $\left|\Pi_{1}\right|<750$ and $\Pi_{3} \approx 0.45$, while in [29] $50<\Pi_{1}<800$ and $\Pi_{3} \approx 0.23$, hence in these studies $\left|\Pi_{1}\right|$ spans at most one order of magnitude within a range we are also covering while $\Pi_{3}$ is fixed, smaller than the values we select, and its effect is not assessed. We explore such a wide range of parameter space to capture both the small and large activity range and include both the FDL regime and a range of parameters where the velocity field has the ability to distort the nematic profile. The lower boundary for the $\Pi_{3}=\Gamma \eta$ range is limited by the computational cost of simulations. We have run simulations at least up to a time $T_{\text {final }} \approx \tau=L^{2} / \Gamma K$, sufficient to ensure convergence to a steady state if it exists. We have observed that there is no possibility to reach a steady state for an extensile nematics, $\alpha>0$, at large $\Pi_{1}$ and away from the FDL $\left(\Pi_{3} \ll 1\right)$. Here solutions remain unsteady as marked in Fig. 2(b). We stress that even in the flow-tumbling regime we obtain steady-state profiles as reported in [13] rather than oscillatory solutions, as, for example, in [30].

In Figs. 2(a) and 2(b), the large $\Pi_{3}$ and low $\left|\Pi_{1}\right|$ region where the solution is given to a very good approximation by Eq. (12) is conveniently identified by a vanishing small magnitude. Outside this area the numerical solution deviates from (12) differently for negative and positive $\alpha$. In particular, for large negative values of the $\Pi_{1}$ parameter the flow is suppressed, while for large positive values the behavior becomes unsteady. For positive intermediate values of $\Pi_{1}$, large velocities develop as signaled in the right panel by a dark blue band that bends toward larger $\Pi_{3}$ for larger $\left|\Pi_{1}\right|$. Qualitatively these solutions correspond to those reported in Figs. 14 and 15 in [13].

Figures 2(c) and 2(d) show how the velocity profiles change with $\Pi_{3}$ for a fixed negative and positive value of the $\Pi_{1}$ parameter. For negative $\alpha$ the rescaled velocity magnitude decreases with $\Pi_{3}$, while in parallel the velocity profile becomes more and more asymmetric: the positive peak moves toward the wall with parallel anchoring, while the negative peak flattens; the trend continues until for the smallest $\Pi_{3}$ the velocity vanishes. For positive $\alpha$ the profile changes as $\Pi_{3}$ decreases, from the analytical result, Eq. (12), to an either entirely positive or negative one of larger magnitude (the sign is randomly selected by the system); in this configuration, the peak is roughly located in the middle of the domain. As $\Pi_{3}$ is further decreased, the rescaled velocity magnitude is reduced, sharper, and multiple peaks appear until the profile becomes unsteady. To provide an overall view on the structure of the active nematics, panels (e)-(g) in Fig. 2 represent the director field in the channel for three calculations of map (a) and (b) as indicated by the plot titles. Case (e) corresponds to the analytical solution, Eq. (11).

We have verified the sensitivity of the steady-state solutions to different initial conditions by repeating the calculations of Fig. 2 with different initial $\mathbf{n}$-profiles as detailed in the caption of 
$\log _{10}\left(\max \left|u_{x}\right| / \max \left|u_{x, \text { analytical }}\right|\right)$

(a)
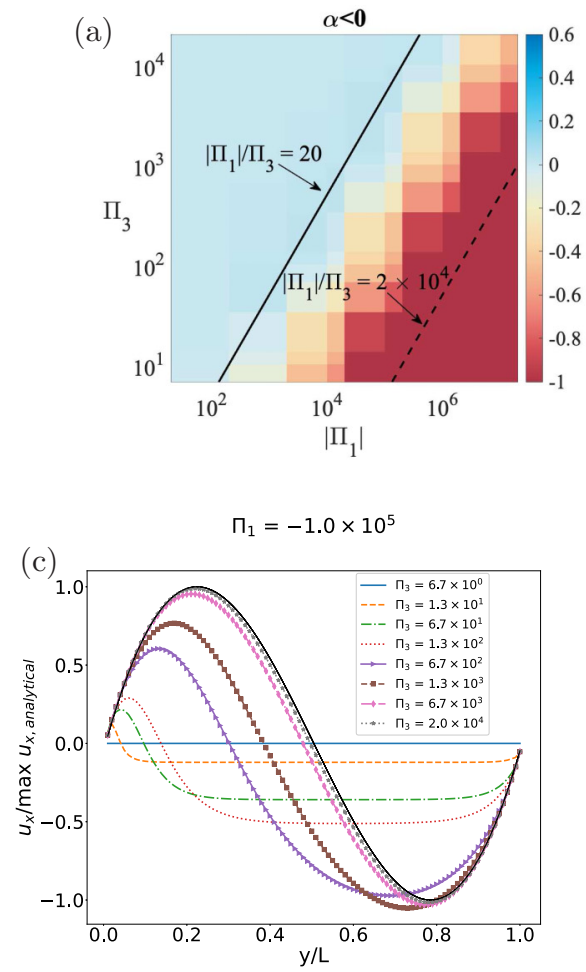

(e)

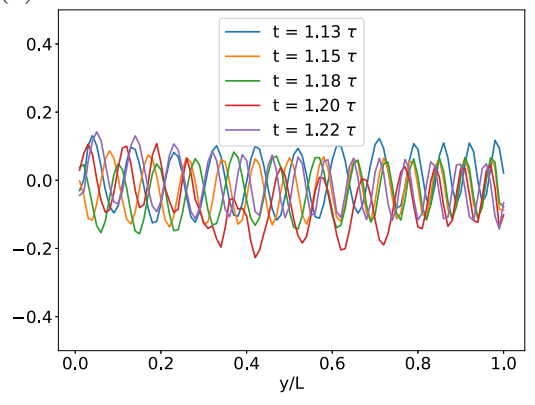

(b)

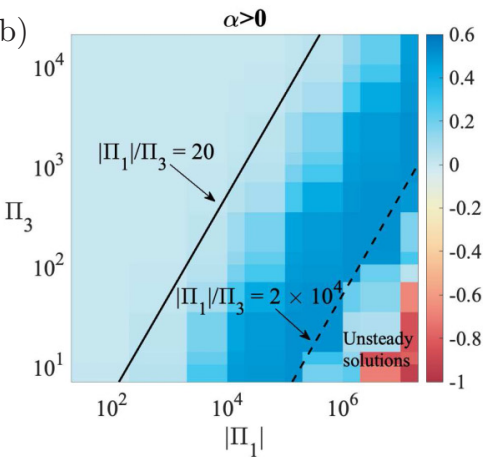

(d)
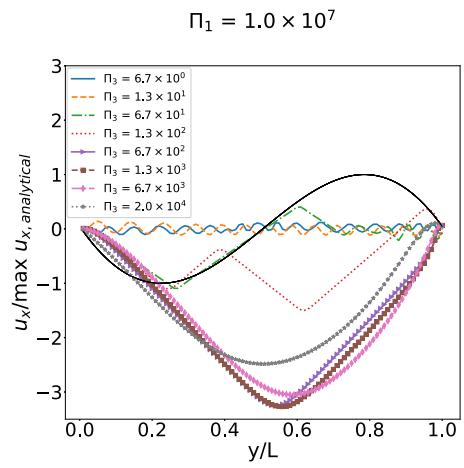

(f)

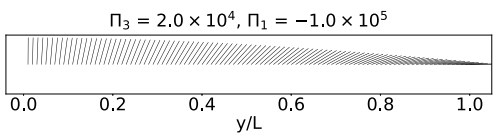

(g)

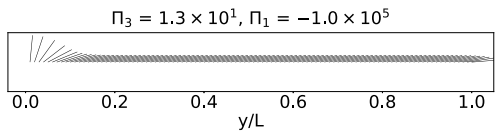

(h)

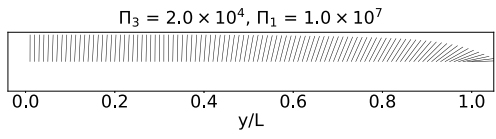

FIG. 2. Top: Base 10 logarithm of the maximum magnitude of the velocity computed numerically and rescaled by the maximum of the analytical profile (12) for a contractile (a) and extensile (b) activity parameter, $\alpha$. This normalized velocity is plotted as a function of $\Pi_{3}$ and $\left|\Pi_{1}\right|$ for a flow-aligning nematics $(\xi=0.7)$. The axes are in logarithmic scale, and the map reports results for a total of 330 separate calculations. Simulations with smaller values of $\Pi_{3}$ are more demanding in computational terms given the slower convergence: for our choice of parameters, the slowest calculations run for $9.8 \times 10^{8}$ time steps. Middle: rescaled velocity profiles corresponding to cases that lie on a vertical cut of the color maps in (a) and (b) as specified by the legend and title of the plot for a negative (c) and positive (d) value of the activity parameter. In (d) the flow profiles with $\Pi_{3}<6.67 \times 10^{2}$ are unsteady. In these cases we display the configuration at the final time $T_{\text {fin }}$. We label as unsteady those calculations for which the RMS deviation in the last 10 saved time steps spaced by approximately $\tau / 50$ time units is below $0.25 \%$. The thick black curves correspond to the analytical solution (12). In (e) we show some intermediate configurations for a selected unsteady case. Bottom: (f)-(h) director field orientation associated with three cases as detailed by the plot titles. 
(a)

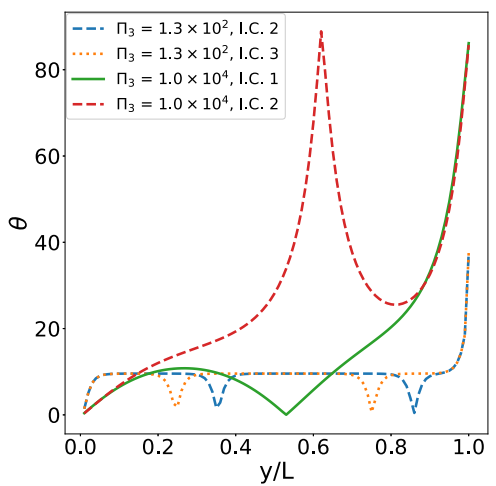

(b)

$$
\Pi_{1}=1.0 \times 10^{7}
$$

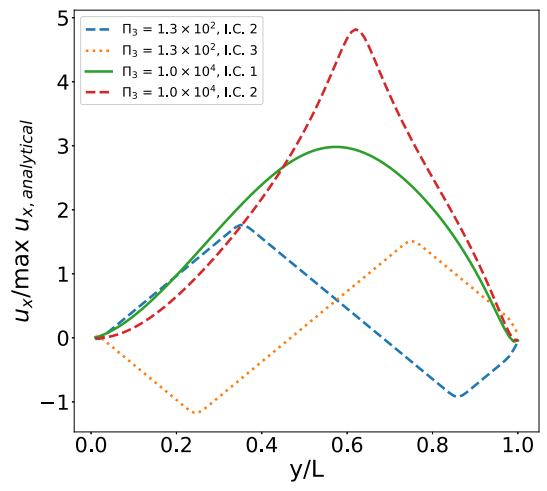

FIG. 3. Nematic angle $\theta=\arctan \left(n_{y} / n_{x}\right)$ (a) and velocity profile (b) for two of the calculations reported in Figs. 2(b) and 2(d) and additional simulations performed for the same values of the model parameters and different initial conditions (I.C.). In the legend, "I.C. 1" corresponds to the initial condition of Fig. 2, that is, an $n_{x}=1$ field perturbed by random noise, "I.C. 2 " is given by $n_{x}=\cos (\pi y / L), n_{y}=\sin (\pi y / L)$, while "I.C. 3 " is Eq. (11). In all cases, the velocity field is initialized to zero.

Fig. 3. We find some dependence on the initialization for the $\alpha>0$ solutions with values of the parameters that lie in the parameter-space region located in between the FDL and the unsteady solutions; see Fig. 3 for two representative examples. As expected, no dependence on the initial conditions is found in the FDL region, as well as in the $\alpha<0$ semiplane of parameter space.

The results reported in Fig. 2 are obtained for a flow-aligning nematic, $\xi=0.7$. For the flowtumbling regime, e.g., $\xi=0.3$, the results differ: the velocity displays a behavior similar to panel (a) of Fig. 2 for both positive and negative values of the activity parameter, and the rescaled velocity profiles vary with $\Pi_{3}$ similarly to panel (c) of Fig. 2, see Figs. 4(a)-4(d). More precisely, although even for the flow-tumbling case there are quantitative differences between the results for a negative and positive activity parameter evident by comparing panels (a) and (b) of Fig. 4, qualitatively an increment in the magnitude of activity or a decrease in $\Pi_{3}$ leads to a suppression of the flow field. Similarly to the flow-aligning case, we also note some instabilities of the numerical solution for large positive values of the activity parameter in the bottom right corner of Fig. 4(b). An interpretation of the differences between the flow-aligning and flow-tumbling case is provided in the following section, Sec. III C.

For both the flow-aligning and flow-tumbling case, the effect of decreasing the $\Pi_{3}$ parameter is similar to that of increasing the $\left|\Pi_{1}\right|$ parameter, hence for both positive and negative values of activity the smooth transition from the frozen director limit regime occurs along lines of constant $\left|\Pi_{1}\right| / \Pi_{3}=|\alpha| L^{2} /(\Gamma \eta K)$. We draw two of them in Figs. 2(a) and 2(b) and Figs. 4(a) and 4(b): one for $\left|\Pi_{1}\right| / \Pi_{3}=20$ marking the deviation from solution (12), and one for $\left|\Pi_{1}\right| / \Pi_{3}=2 \times 10^{4}$ signaling a second transition to the zero velocity or the unsteady behavior for $\alpha>0$. Given the relevance of the $\Pi_{1} / \Pi_{3}$ dimensionless group, we will from now on refer to it with the new symbol $\Phi:=\Pi_{1} / \Pi_{3}$. Note that in Figs. 4(c) and 4(d) we report the velocity profiles for calculations that in Figs. 4(a) and 4(b) maps lie along a line of maximum variation of $|\Phi|$, that is, a line perpendicular to the $|\Phi|$ isolines, rather than on a vertical cut as in Figs. 2(c) and 2(d). We have verified in Fig. 5 that for the same value of $|\Phi|$ we obtain the same director field profile $\mathbf{n}(y)$, and the velocity profiles collapse on a single curve provided that they are rescaled by the activity parameter $\alpha$. In conclusion, we have hypothesized in Sec. II B that solutions would depend on three parameters: $\Pi_{1}, \Pi_{3}$, and $\xi$, and we have found numerically that results practically depend on two parameters $\Phi$ and $\xi$.

For the flow-tumbling case, the profiles for the positive/negative $\Phi$ appear flipped left to right, and top to bottom. We will provide an explanation for this in Sec. III C. Note that since we have 
$\log _{10}\left(\max \left|u_{x}\right| / \max \left|u_{x, \text { analytical }}\right|\right)$
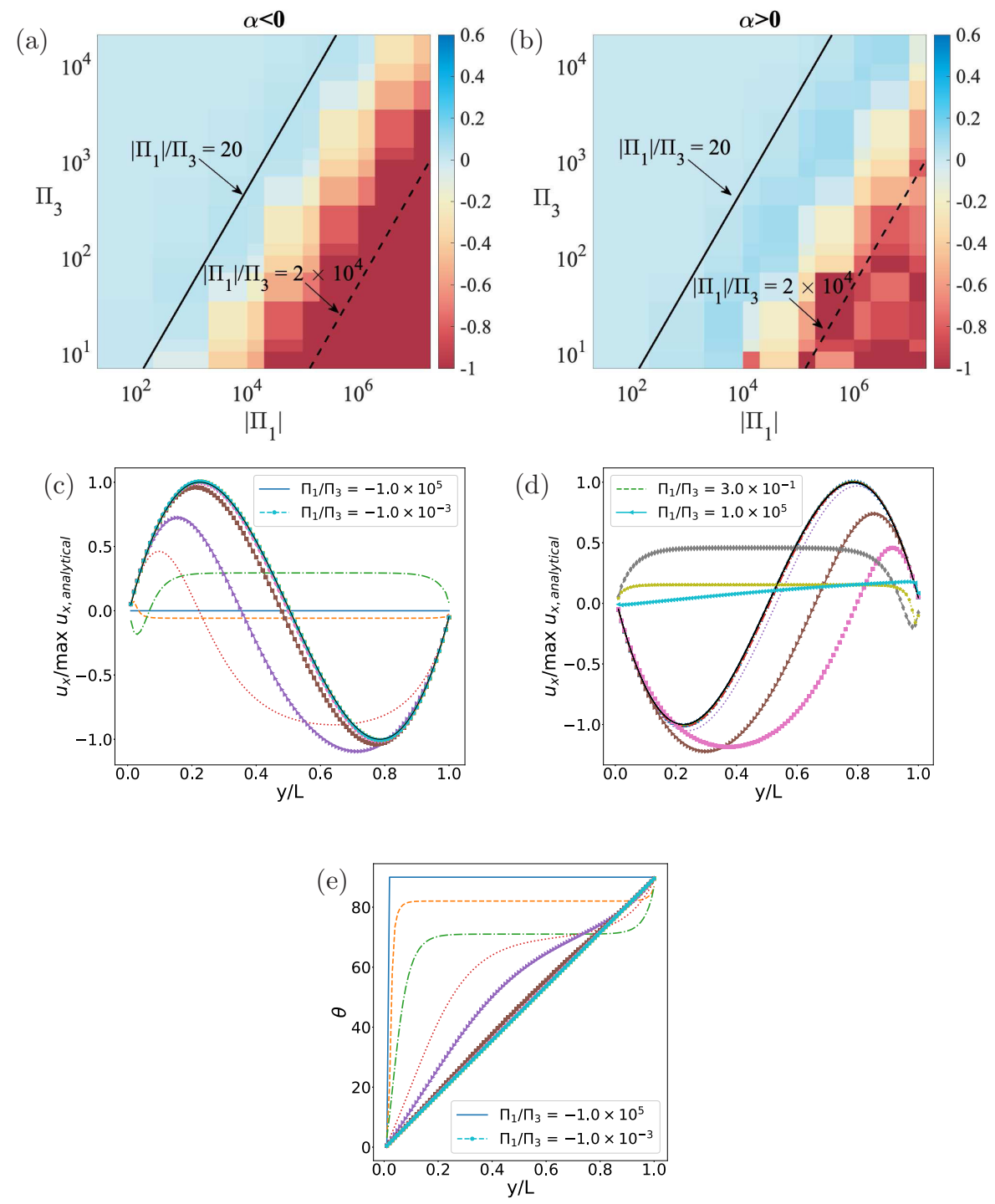

FIG. 4. Top: Base 10 logarithm of the maximum magnitude of the velocity computed numerically and rescaled by the maximum of the analytical profile (12) for negative (a) and positive (b) values of the activity parameter and a flow-tumbling nematics $(\xi=0.3)$. The axes correspond to the $\Pi_{3}$ and $\left|\Pi_{1}\right|$ parameters and are in logarithmic scale. Two $\left|\Pi_{1}\right| / \Pi_{3}$ isolines are shown in panels (a),(b): $\left|\Pi_{1}\right| / \Pi_{3}=2 \times 10^{4}$ (dashed-black line) and $\left|\Pi_{1}\right| / \Pi_{3}=20$ (solid-black line). Middle: rescaled velocity profiles corresponding to calculations that lie on a diagonal cut of the color maps in (a) and (b); the cuts originate at the top-left corner of the maps and run perpendicular to the $\left|\Pi_{1}\right| / \Pi_{3}$ isolines. For clarity, the legend only labels the two curves that correspond to the extreme values of $\Pi_{3} / \Pi_{1}$. The thick black lines correspond to the analytical solution (12). Observe the remarkable resemblance of the rescaled velocity profiles in (c) with those reported in Fig. 2(c) for the flow-aligning case. Bottom: nematic angle $\theta=\arctan \left(n_{y} / n_{x}\right)$ corresponding to the rescaled velocity profiles in (c). The analytical solution corresponds to a straight line, while the zero-flow solution corresponds to a discontinuous profile that suddenly jumps close to the bottom wall from $n_{x}=1$ to $n_{y}=1$; this is allowed in the $\mathbf{Q}$-model by a concomitant $q_{0}=0$. 

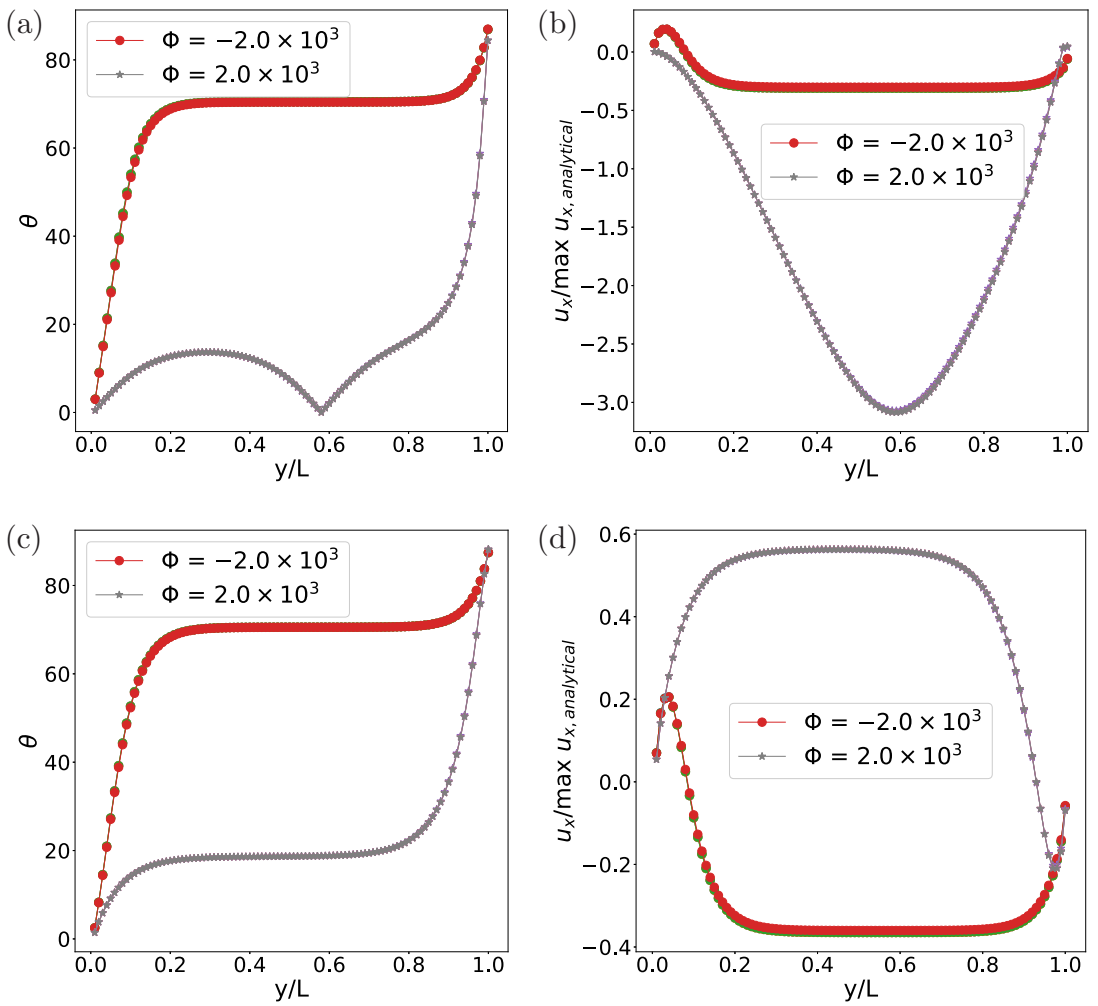

FIG. 5. Nematic angle $\theta=\arctan \left(n_{y} / n_{x}\right)$ (a),(c) and velocity profile (b),(d) rescaled by the maximum of the analytical profile for the flow-aligning case (a),(b) and the flow-tumbling case (c),(d) and two different values of the $\Phi=\Pi_{3} / \Pi_{1}$ dimensionless group.

collapsed two dimensionless parameters into one, this allows us to reproduce the solutions in Figs. 14 and 15 of [13] using larger $\Pi_{3}$ values if this is compensated by smaller $\left|\Pi_{1}\right|$ and if the remaining dimensionless numbers, specifically $\Pi_{2}$, are the same. This reduction of parameter space also explains why in Sec. III A 2 solution (12) was found to a very good approximation up to $\left|\Pi_{1}\right|=10^{3}$, in that specific case in fact $\Pi_{3} \approx 10^{4}$ making the $\left|\Pi_{1}\right|=10^{3}$ threshold equivalent to $\Phi \approx 0.1<1$.

As a final observation we note a qualitative similarity between the velocity profiles for a contractile nematics for both the flow-tumbling and -aligning regime; compare Figs. 2(c) and 4(c). This suggests that those solutions may have only a weak dependence of the flow-aligning parameter $\xi$.

\section{Interpretation of results}

A stability analysis performed on the n-model [8] and later results [10] built on expanding concepts presented in [31] show that for a 1D slab geometry in a flow-aligning regime, a nematic profile parallel to the walls is (i) unstable for extensile active particles $(\alpha>0)$ and (ii) stable for contractile ones $(\alpha<0)$. Similarly, a nematic arrangement perpendicular to the walls is (iii) unstable for extensile active particles and (iv) stable for contractile ones. In these cases, the instability appears above a certain activity threshold $\alpha_{c}$ that depends on several model parameters: the system size $L$, the dynamic viscosity of the flow $\eta$, and the elastic, flow-aligning, and rotational diffusivity parameter; see, e.g., [10] for an analytical expression for $\alpha_{c}$. We have verified numerically that this critical threshold also predicts the transition to spontaneous active flows in the $\mathbf{Q}$-model when 


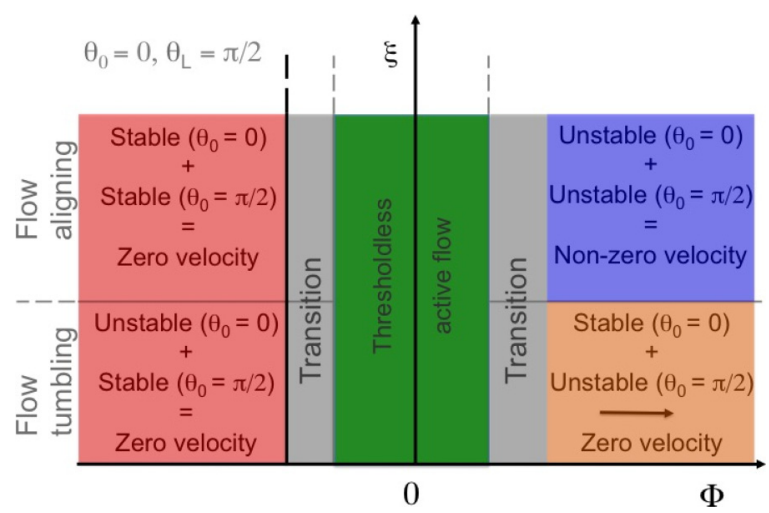

FIG. 6. Schematic representation of the numerical results reported in Sec. III B. For small values of the dimensionless parameter $\Phi=\Pi_{1} / \Pi_{3}$ we observe thresholdless active flows [14] independently of the sign of the activity parameter or the value of the flow-aligning parameter (green shaded area). For intermediate values of $|\Phi|$ the velocity field is nonzero and depends on the sign of the activity parameter as well as the value of the flow-aligning parameter (gray shaded area). There exists a very close resemblance of the velocity profiles in the transition region for three cases over four: positive activity and flow tumbling Fig. 4(d), negative activity and flow tumbling Fig. 4(c), negative activity and flow aligning Fig. 2(c). The positive activity and flow-aligning case differs and shows some dependence on the initial conditions, Fig. 2(d). The behavior in the regions of large magnitude of $\Phi$, beyond the transition regions, can be rationalized on the basis of previous studies [8,10,31] for the flows of active nematics in channels with either homeotropic or parallel boundary conditions. The hybrid boundary condition $\left(\theta_{0}=0, \theta_{L}=\pi / 2\right)$ can be interpreted as a combination of parallel and perpendicular boundary conditions. For those cases, the stability conditions have been derived in the literature, and their combination suggests the type of flow we observe in the hybrid case (blue and red shaded areas).

the anchoring is forced through Dirichlet boundary conditions $n_{x}=\cos \theta, n_{y}=\sin \theta$. In [10] free boundary conditions were imposed for the director field at the walls $\left(\partial_{y} n_{i}=0\right)$.

Hybrid boundary conditions can be viewed as a combination of the four scenarios (i)-(iv). Both nematic arrangements, parallel and perpendicular to the walls, are unstable and will result in a nonzero flow for positive and large enough $\alpha$, while instabilities are suppressed for both these configurations when $\alpha<0$. This explains why a deviation from the FDL will lead for $\alpha<0$ to a suppression of the flow and for $\alpha>0$ to a nonzero velocity profile providing an explanation for the behavior reported in Figs. 2(a) and 2(b). More specifically, the analogy with the homogeneous director field can be thought to hold locally according to the mechanism described in [31]. In contractile systems, both the parallel and perpendicular nematic arrangements are stable. Therefore, if in the neighborhood of the walls where the anchoring is fixed the nematic profile is distorted from the parallel or perpendicular alignment, such distortions will die away. Two separate domains will form, one with uniform $n_{x}=1$ and one with uniform $n_{y}=1$, and a discontinuity in the $\mathbf{n}$ profile will appear where $q_{0}$ will modulate its magnitude and go to zero; see Sec. IIIC 1. See Fig. 6 for a schematic representation of our interpretation of the results.

The situation is different for the flow-tumbling regime: in this case a nematic arrangement parallel to the walls is stable for extensile active particles and unstable for contractile ones. On the contrary, a nematic arrangement perpendicular to the walls is unstable for extensile active particles and stable for contractile ones [10]. Hence mixed boundary conditions in the flow-tumbling regime correspond to the combination of a stable and unstable configuration in which the stable tendency wins over the unstable one leading to a zero-velocity profile as the magnitude of activity increases; see the schematic representation in Fig. 6.

For intermediate values of the activity parameter in the flow-tumbling regime, the velocity profiles for $\alpha>0$ closely resemble the velocity profiles for the $\alpha<0$ case once "flipped" about 


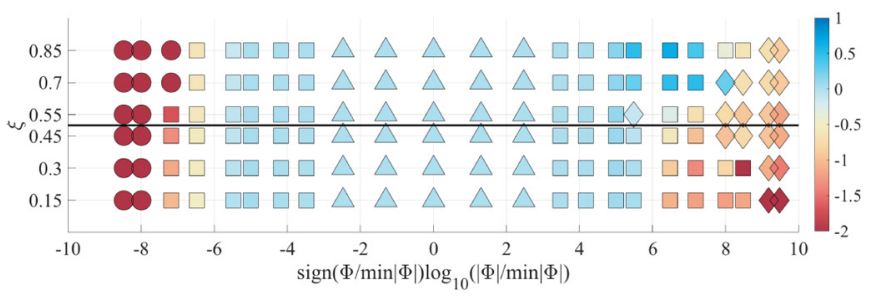

FIG. 7. Map of the numerical solutions for the 1D velocity profile as a function of the flow-aligning parameter $\xi$ and the dimensionless number $\Phi$. Different symbols correspond to different types of solutions: circles denote zero-velocity solutions that correspond to a diagonal $\mathbf{Q}$-tensor; triangles indicate solutions whose normalized root-mean-square (RMS) value does not deviate from Eq. (12) by more than 0.01; squares indicate all the other types of solutions, and diamonds designate unsteady solutions. The color represents the rescaled magnitude of velocity as in Figs. 2(a) and 2(b) and Figs. 4(a) and 4(b). The boundary between the flow-tumbling and flow-aligning behavior is given by $\xi=0.5$ and is marked by a black line. Note the expression for the $x$-axis chosen to display $\Phi$ in log-scale while distinguishing between the positive and negative cases.

the $y$-axis; this symmetry reflects the symmetries embodied in the equations for the nematic field as stressed in [10]: a change in the sign of $\alpha$ is equivalent to a change in sign of the flow-aligning parameter in conjunction with a $\pi / 2$ rotation of the director field. Therefore, changing the sign of $\alpha$ in our setting is equivalent to exchanging the $y=0$ and $y=L$ boundary conditions as emerges also from Figs. 5(c) and 5(d).

Figure 7 displays on the $\xi-\Phi$ plane the different types of solutions described in this work for the same $\Phi$ values of Figs. 2, 4, and some additional $\xi$ values. This plot corresponds to the numerical outcome and corroborates the schematic representation of Fig. 6.

\section{Further remarks on the zero-flow solution}

The nematic profile selected dynamically by the system and associated with the zero-flow steady state is a free-energy stationary point that satisfies $\mathcal{H}_{i j}=0$ and corresponds to a zero-curl active force. This second condition is verified in our setting anytime the off-diagonal terms of the $Q_{i j}$ tensor are zero. The nematic profile will therefore satisfy an undamped unforced Duffing equation: $K Q_{x x}^{\prime \prime}-A Q_{x x}-2 C Q_{x x}^{3}=0$ in $2 \mathrm{D}$ and the system of nonlinear ODEs,

$$
\begin{aligned}
& Q_{x x}^{\prime \prime}=\frac{1}{K}\left[A Q_{x x}+B Q_{x x}^{2}+2 C\left(Q_{x x}^{2}+Q_{y y}^{2}+Q_{x x} Q_{y y}\right) Q_{x x}-2 B\left(Q_{x x}^{2}+Q_{y y}^{2}+Q_{x x} Q_{y y}\right) / 3\right], \\
& Q_{y y}^{\prime \prime}=\frac{1}{K}\left[A Q_{y y}+B Q_{y y}^{2}+2 C\left(Q_{x x}^{2}+Q_{y y}^{2}+Q_{x x} Q_{y y}\right) Q_{y y}-2 B\left(Q_{x x}^{2}+Q_{y y}^{2}+Q_{x x} Q_{y y}\right) / 3\right]
\end{aligned}
$$

in 3D. The solutions obtained with hybrid anchoring boundary conditions for $\Pi_{2} \ll 1$ are characterized by sharp fronts in the $q_{0}$ profile where $q_{0} \rightarrow 0$ while $\mathbf{n}$ changes orientation to match the boundary conditions switching from $n_{x}=1, n_{y}=0$ to $n_{x}=0, n_{y}=1$ [see Fig. 4(e), blue curve]. In the 3D case, biaxiality develops in the region where $\mathbf{n}$ changes orientation (see Sec. IIIE). These solutions reflect the greater generality of the $\mathbf{Q}$-model, in fact they are not admitted in the $\mathbf{n}$-model where the magnitude of the nematic order parameter is fixed. In conclusion, we discover that in addition to Eq. (11), which we refer to as fixed point 1 (FP1), the Euler-Lagrange equation for the Q-model admits a second stable fixed point, FP2, that allows for steady-state zero-flow solutions that manifest at nonzero activity. In the following section, we deepen our analysis of these two configurations.

\section{Comments on the minimum-energy solutions in the $\mathbf{Q}$ model}

In Sec. III A 2 the numerical solution for a 3D-Q tensor, FP1, was obtained for values of the thermotropic constants and $q_{0}$ that corresponded to minimum-energy solutions for uniform states 


$$
\mathrm{CL}^{2} / \mathrm{K}=60000
$$
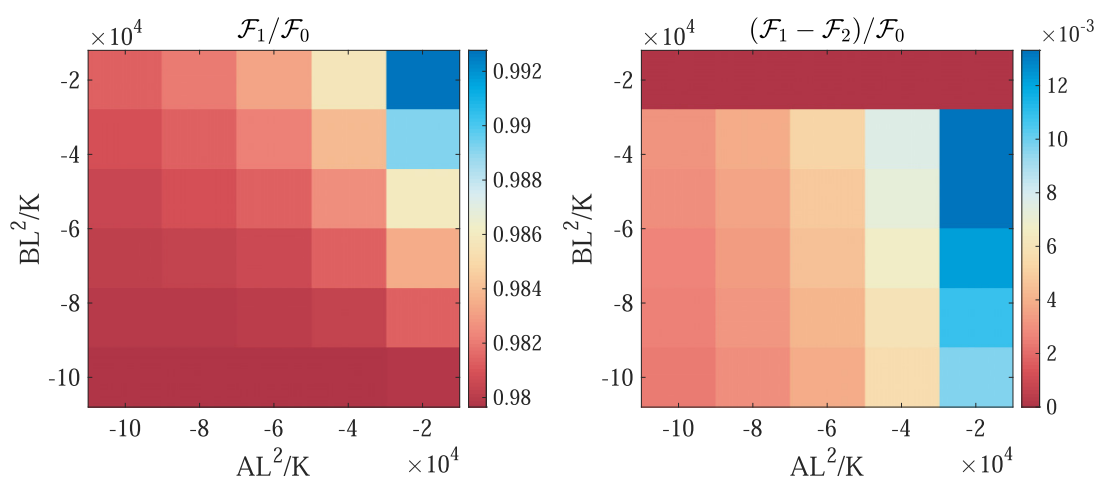

FIG. 8. (a) Rescaled Landau-de Gennes free energy as a function of the dimensionless parameters $B L^{2} / K$ and $C L^{2} / K$ for the solution of $\mathcal{H}_{i j}=0$ obtained by relaxing the initial condition IC1 through Eq. (23). The free energy is rescaled by a reference free-energy value, $\mathcal{F}_{0}$, corresponding to a homogeneous solution for a system of size $L$. (b) Difference between the Landau-de Gennes free energy associated with IC1, $\mathcal{F}_{1}$, and IC2, $\mathcal{F}_{2}$, rescaled by $\mathcal{F}_{0}$. These calculations have been performed for $C L^{2} / K=60000$ and repeated for $0<C L^{2} / K<$ 100 000. In this parameter range, we observe qualitatively the same type of solutions; differences concern the magnitude of $q_{0}$ in a narrow region close to the boundaries.

( $q_{0}=$ const); see the caption of Fig. 1. These same values were used when integrating the full set of equations leading to the dynamical selection of FP2 for low $\Phi$. We now test the sensitivity of the two fixed points to the parameters $A, B$, and $C$, by looking for solutions of $\mathcal{H}_{i j}=0$ in a neighbourhood of the previously selected values: we vary $A$ and $B$ in the range $-1.67 \leqslant A / C \leqslant 1.67$ and $-1.67 \leqslant B / C \leqslant 0.0$ with $C L^{2} / K=6 \times 10^{4}$. We always constrain the choice of parameters to thermodynamically stable states $(C>0)$ [32]. Numerically, we find minimum-energy solutions relaxing the order parameter through Eq. (23) with fixed anchoring at the walls. We repeat the calculations for two different initial conditions: expression (11), referred to as "IC1," and a discontinuous initial state with $n_{x}(y)=1$ for $y=[0, L / 2), n_{y}(y)=1$ for $y=[L / 2, L]$, IC2. As expected [24], the solution converges to a nematic state for $A<0$ and an isotropic state for $A>0$. The isotropic state is only attained in the middle of the domain given the fixed anchoring at the walls. For $B / C<0$ and IC1 the nematic solution corresponds to a nematic state with a nonzero curl active force of the kind reported in Sec. III A 2: the nematic profile corresponds to Eq. (11) to a very good approximation while the $q_{0}$ profile slightly changes as a function of the thermotropic parameters. In Fig. 8(a) we show the free energy of this solution as a function of the thermotropic parameters, and we find that when compared to a uniform state solution, the most energetically favorable configurations are attained for the largest $A L^{2} / K$ and $B L^{2} / K$. For $B / C<0$ and IC2 the solution is a nematic state with a zero-curl active force and corresponds to FP2. For the special case $B=0$ both IC1 and IC2 converge to FP2. When we compare the free-energy value for the solutions obtained with IC1 and IC2, Fig. 8(b), we find that FP2 has the largest energy, hence it is a local minimum.

\section{E. An example of biaxial thresholdless active flows in the $Q$ model}

The Landau-de Gennes free energy adopted in the Q-model [Eq. (2)] allows for a wider family of minimum-energy solutions than the Franck free energy used in the $\mathbf{n}$-model because it includes a thermotropic term in addition to a distortion term. This fact is relevant when dealing with thresholdless active flows since they require minimum-energy nematic profiles with a nonzero curl active force. Potentially, the $\mathbf{Q}$-model allows for more thresholdless active flow configurations than 
(a)

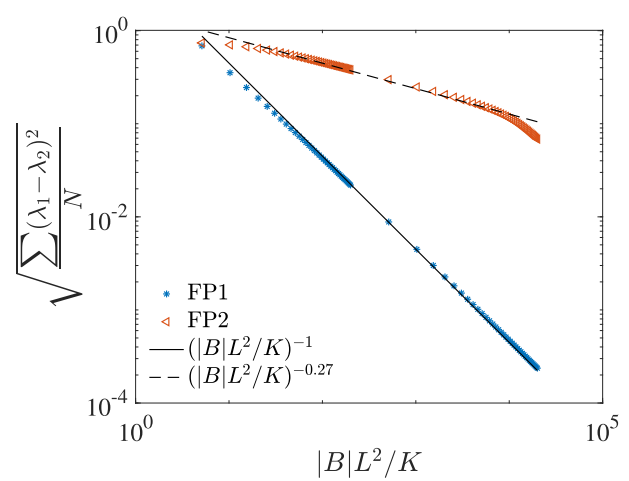

(c)

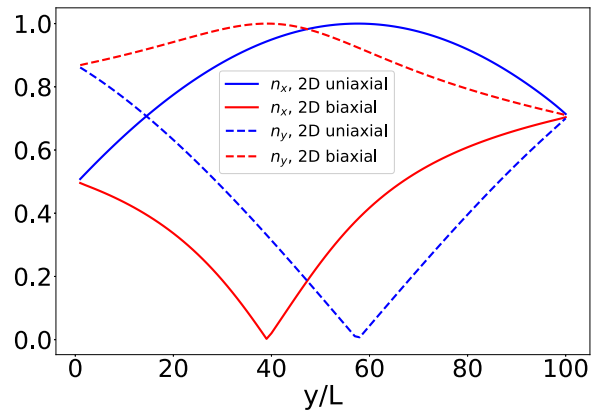

(b)

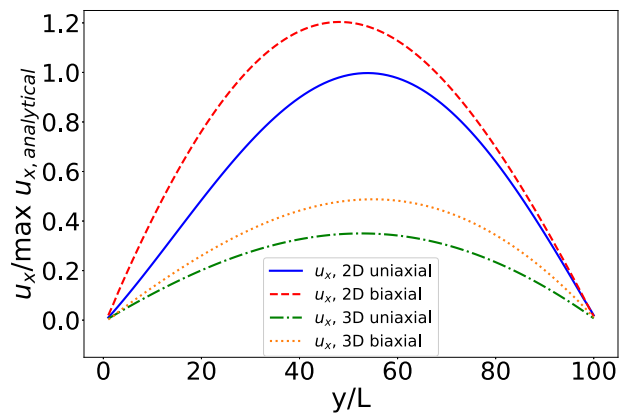

(d)

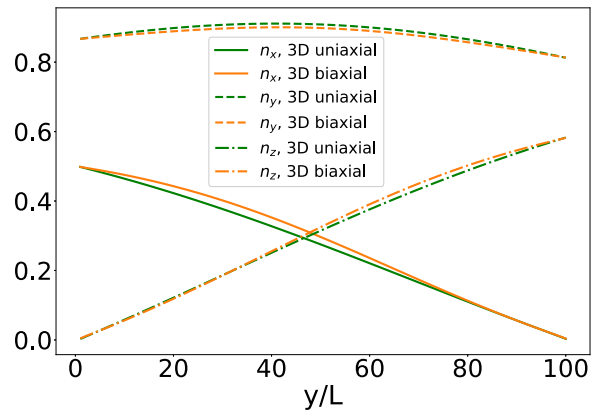

FIG. 9. (a) Root-mean-square deviation from uniaxiality measured in terms of the difference between the closest eigenvalues of the $Q_{i j}$ tensor for FP1, FP2, and $B L^{2} / K \rightarrow 0$, here $C L^{2} / K=|A| L^{2} / K=60000$. The solid and dashed lines represent power-law scalings as reported in the legend. (b) Velocity profile for a thresholdless active flow for a 2D and 3D geometry and for a uniaxial case (ground state of type 3) and a biaxial case (ground state of type 4). The 2D uniaxial profile is given by Eq. (22); the 3D velocity field has a nonzero $u_{z}$ component that is not reported in the plot for clarity. All the curves are rescaled by the maximum of the analytical profile (22). In panels (c) and (d) we report the nematic director field for the 2D (c) and 3D (d) solution.

the n-model, including, in 3D, biaxial solutions. The objective of this section is to identify some of them.

To decide whether the degree of biaxiality of a solution is non-negligible, a threshold is set on the difference between the two closest eigenvalues $\lambda_{i}, \lambda_{j}$ of tensor $\mathbf{Q}$ : if $\sqrt{\sum_{k}\left(\lambda_{i}-\lambda_{j}\right)^{2}} / L>10^{-4}$, where $L$ is the system size and $k$ are the grid points, then biaxiality is considered non-negligible.

We classify the type of minimum-energy solutions that we obtain in five categories: isotropic states (type 0), nematic states with a zero-curl active force $\nabla \times f_{a}=0$ and negligible biaxiality (type 1), biaxial nematic states with a zero-curl active force (type 2), nematic states with a nonzero curl active force $\nabla \times f_{a} \neq 0$ and negligible biaxiality (type 3 ), and biaxial nematic states with a nonzero curl active force (type 4). Only solutions of type 3 and 4 can support thresholdless active flows. The two fixed-point solutions discussed so far correspond to type 3, FP1, and type 2, FP2. A closer inspection of FP1 reveals that this fixed-point solution has non-negligible biaxiality in the neighborhood of $B L^{2} / K=0$. Similarly, the biaxial fixed point FP2 more markedly deviates from a uniaxial arrangement as $B L^{2} / K \rightarrow 0$; see Fig. 9(a). The RMS deviation from biaxiality for both FP1 and FP2 follows a power law as reported in Fig. 9(a).

The only biaxial solution identified so far for $B L^{2} / K=0$, FP2, will not be able to sustain a thresholdless active flow, however different anchoring choices will change this picture. For example, for anchoring angles of $60^{\circ}$ and $45^{\circ}$ and $B=0$, the ground state is biaxial and has a nonzero curl 
active force, therefore it supports a biaxial thresholdless active flow, Figs. 9(b) and 9(d). If this geometry is extended in 3D, meaning that the plane formed by the anchoring angles at the walls is not orthogonal to the walls [the angle $\theta(0)$ lies on the $x-y$ plane, the angle $\theta(L)$ lies on the $y-z$ plane], the picture is similar, Figs. 9(a) -9 (d). Note that for $B=0$ the isotropic-nematic phase transition is second order instead of first order, and consider that the degree of biaxiality grows as the value of the $B$ parameter approaches zero, Fig. 9. We can therefore conclude that biaxiality is relevant for a weakly first-order isotropic-nematic phase transition.

\section{CONCLUSIONS}

We study active nematic flows confined in a quasi-one-dimensional channel geometry with hybrid alignment at the walls. More specifically, we impose a fixed anchoring parallel to one wall and perpendicular to the second. Active flows in this setting have been investigated in previous studies revealing interesting features: in [13] it was shown how small positive and negative values of the activity parameter lead to different velocity profiles, while in [14] it was demonstrated that this geometry presents a nonzero velocity field even for vanishingly small values of the activity parameter. In [14] an analytical solution for such a thresholdless active flow was derived within the active nematohydrodynamic n-model for small activity and in the frozen director limit (FDL), that is, in a regime where the nematic is not distorted by the flow and satisfies the Euler-Lagrange equation for minimizing the free energy. In this paper, we have shown that this solution holds also in the active nematohydrodynamic $\mathbf{Q}$-model for a two-dimensional $\mathbf{Q}$-tensor, a result that can be generalized to any anchoring angle. We reproduce this solution numerically with a hybrid lattice-Boltzmann code identifying the range of model parameters for which this result is found with high accuracy. In addition, we verify numerically that this nematic and velocity profile is a very good approximation of the solution for a three-dimensional Q-tensor.

The active nematohydrodynamic $\mathbf{Q}$-model generally depends on six dimensionless numbers. However, in our specific geometry, in the absence of an external forcing, and for system sizes much larger than the characteristic defect core, we expect the solution to depend on three dimensionless groups: $\Pi_{1}$, which is the square of the ratio between the active length scale and the size of the system, $\Pi_{3}$, a parameter that measures the distance from the FDL regime identified by $\Pi_{3} \gg 1$, and the flow aligning parameter $\xi$ that expresses the tendency of particles to tumble or align with the flow. With the aim of providing a unifying picture for active flows in a channel with hybrid anchoring at the walls, we have computed numerically steady-state solutions in a wide portion of parameter space: the parameter $\left|\Pi_{1}\right|$ spans six orders of magnitude, the parameter $\Pi_{3}$ spans almost four orders of magnitude, while the values of $\xi$ encompass both the flow-tumbling and -aligning regime. These parameter ranges include both the FDL and a parameter region where the velocity field has the ability of distorting the nematic profile, and they comprise both the small and large activity limit. Our study reveals that the effect of decreasing $\Pi_{3}$ is similar to that of increasing $\left|\Pi_{1}\right|$ so that the transition from the FDL solution occurs along lines of constant $\Phi=\Pi_{1} / \Pi_{3}$, hence the solution only depends on two dimensionless groups: $\Phi$ and $\xi$, a result that could not be anticipated theoretically.

We observe that the symmetric thresholdless active flow derived in [14] manifests to a very good approximation for small values of $\Phi$ and is independent of the sign of activity and the value of $\xi$. Moving away from the low $\Phi$ region, the transition from the symmetric active flow is smooth with the model parameters and depends on them. In particular, for (i) a flow-aligning and contractile nematic, the velocity profile becomes more and more asymmetric while its magnitude rescaled by the activity parameter decreases as $|\Phi|$ increases until the flow is completely suppressed; for (ii) a flow-aligning and extensile nematic, the velocity profile loses symmetry as $\Phi$ increases until it becomes unsteady. For intermediate values of the parameter, the velocity profile has a single peak located around the middle of the domain. This steady-state configuration displays some dependence on the initial condition for the nematic director field. Unlike in the flow-aligning regime, in the flowtumbling regime the deviation from the FDL profile is similar for a (iii) contractile and (iv) extensile 
nematic; in both cases the profile decreases in relative magnitude until the flow is suppressed as $|\Phi|$ increases.

We interpret the different flow-aligning and tumbling behaviors for large $|\Phi|$ in terms of the stability of simpler configurations with either parallel or perpendicular anchoring at both walls [10]. In the flow-aligning regime, both a parallel and a perpendicular configuration is stable to perturbations for negative activity and unstable for positive activity. This provides a rationale for the zero-flow solution observed for a contractile nematic with hybrid anchoring at the walls and the nonzero large magnitude or unsteady velocity solution found for an extensile nematic with hybrid anchoring. In the flow tumbling regime, the picture is different. For a contractile particle, the flow is unstable to perturbation for a parallel arrangement and stable for a perpendicular one, while the opposite is true for extensile active particles. This means that mixed boundary conditions correspond to a combination of a stable and unstable configuration for both negative and positive activity, and we observe that the stable tendency wins over the unstable one leading to zero-flow solutions qualitatively very similar to the zero-flow solutions found for the contractile flow-aligning case. Therefore, unlike in the flow-aligning case, in the flow-tumbling case there is a symmetry in the behavior for positive and negative values of activity.

In the zero-flow configuration, the nematic director $\mathbf{n}$ reorients abruptly from $n_{x}=1, n_{y}=0$ to $n_{x}=0, n_{y}=1$ to match the boundary conditions while $q_{0}$ decreases to zero in correspondence with the discontinuity. We clarify that this configuration supports a zero-flow steady state because it corresponds to a local minimum of the free energy and a zero-curl active force. We have found a second stationary point for the free energy in addition to the nematic profile responsible for the thresholdless active flow. This stationary point is a local minimum of the Euler-Lagrange equation and displays biaxiality for a three-dimensional Q-tensor.

Finally, we exploit the greater generality of the $\mathbf{Q}$-model compared to the $\mathbf{n}$-model and provide an example of a biaxial thresholdless active flow for conflicting anchoring at the walls corresponding to a $60^{\circ}$ and $45^{\circ}$ angle on either a two-dimensional or three-dimensional (out of the plane) geometry. For this configuration, the biaxial thresholdless flow exists also in the special case of a symmetric quartic free-energy expression that corresponds to a second-order isotropic-nematic phase transition. In our examples, we find that biaxiality is relevant for a weakly first-order isotropic-nematic phase transition.

As a concluding remark, we remind the reader that our results have been obtained in a onedimensional domain as representative of two-dimensional channel flows that are uniform along the longitudinal direction. We recall that in a truly two-dimensional system, instabilities can develop in the longitudinal direction due to spontaneous symmetry breaking; see, for example, [29,33]. Therefore, an important underlying question is the range of validity of our analysis when extended to 2D systems. Informed by the results of our study, we expect the critical longitudinal wavelength to depend on two parameters: $\Phi$ and $\xi$. Preliminary results point to the fact that the lower the value of the parameter $\Phi$ is, the more robust is the 1D approximation, or else, the longer is the critical longitudinal wavelength, $\lambda_{x, c}$. Assessing the role of the flow-aligning parameter $\xi$ proves to be more difficult. Addressing the functional form of $\lambda_{x, c}$ is by itself a relevant and complex matter that will be the subject of future studies.

\section{ACKNOWLEDGMENTS}

This project has received funding from the European Union's Horizon 2020 research and innovation programme under the Marie Sklodowska-Curie Grant Agreement No. 754462. I.P. acknowledges support from Ministerio de Ciencia, Innovación y Universidades (Grant No. PGC2018-098373-B-100/FEDER-EU), DURSI (Grant No. 2017 SGR 884), and SNSF (Project No. 200021-175719). C.R. thanks Dr. G. Di Staso and Dr. D. Banerjee for useful scientific discussions and the initial support with the Lattice Boltzmann code. 
[1] D. Saintillan, Rheology of active fluids, Annu. Rev. Fluid Mech. 50, 563 (2018).

[2] P.-G. de Gennes and J. Prost, The Physics of Liquid Crystals (Oxford University Press, New York, 1993).

[3] S. P. Thampi and J. M. Yeomans, Active turbulence in active nematics, Eur. Phys. J.: Spec. Top. 225, 651 (2016).

[4] Y. Hatwalne, S. Ramaswamy, M. Rao, and R. A. Simha, Rheology of Active-Particle Suspension, Phys. Rev. Lett. 92, 118101 (2004).

[5] D. Saintillan and M. J. Shelley, Instabilities and Pattern Formation in Active Particle Suspensions: Kinetic Theory and Continuum Simulations, Phys. Rev. Lett. 100, 178103 (2008).

[6] H. H. Wensink, J. Dunkel, S. Heidenreich, K. Drescher, R. E. Goldstein, H. Lowen, and J. M. Yeomans, Meso-scale turbulence in living fluids, Proc. Natl. Acad. Sci. (USA) 109, 14308 (2012).

[7] T. B. Saw, A. Doostmohammadi, V. Nier, L. Kocgozlu, S. Thampi, Y. Toyama, P. Marcq, C. T. Lim, J. M. Yeomans, and B. Ladoux, Topological defects in epithelia govern cell death and extrusion, Nature (London) 544, 212 (2017).

[8] R. Voituriez, J. F. Joanny, and J. Prost, Spontaneous flow transition in active polar gels, Europhys. Lett. 70, 404 (2005).

[9] I. S. Aranson, A. Sokolov, J. O. Kessler, and R. E. Goldstein, Model for dynamical coherence in thin films of self-propelled microorganisms, Phys. Rev. E 75, 040901(R) (2007).

[10] S. A. Edwards and J. M. Yeomans, Spontaneous flow states in active nematics: A unified picture, Europhys. Lett. 85, 18008 (2008).

[11] T. Sanchez, D. T. N. Chen, S. J. DeCamp, M. Heymann, and Z. Dogic, Spontaneous motion in hierarchically assembled active matter, Nature (London) 491, 431 (2012).

[12] M. Ravnik and J. M. Yeomans, Confined Active Nematic Flow in Cylindrical Capillaries, Phys. Rev. Lett. 110, 026001 (2013).

[13] D. Marenduzzo, E. Orlandini, M. E. Cates, and J. M. Yeomans, Steady-state hydrodynamic instabilities of active liquid crystals: Hybrid lattice Boltzmann simulations, Phys. Rev. E 76, 031921 (2007).

[14] R. Green, J. Toner, and V. Vitelli, Geometry of thresholdless active flow in nematic microfluidics, Phys. Rev. Fluids 2, 104201 (2017).

[15] A. Sengupta, S. Herminghaus, and C. Bahr, Liquid crystals microfluidics: Surface, elastic and viscous interactions at microscales, Liq. Cryst. Rev. 2, 73 (2014).

[16] S. Čopar, Ž. Kos, T. Emeršič, and U. Tkalec, Microfluidic control over topological states in channelconfined nematic flows, Nat. Commun. 11, 3528 (2020).

[17] Ž. Kos and M. Ravnik, Field generated nematic microflows via backflow mechanism, Sci. Rep. 10, 2020 (2020).

[18] Y.-J. Na, T.-Y. Yoon, S. Park, B. Lee, and S.-D. Lee, Electrically programmable nematofluidics with a high level of selectivity in a hierarchically branched architecture, ChemPhysChem Commun. 11, 101 (2010).

[19] T. Ohzono and J. ichi Fukuda, Zigzag line defects and manipulation of colloids in a nematic liquid crystal in microwrinkle grooves, Nat. Commun. 3, 701 (2012).

[20] A. Sengupta, C. Bahr, and S. Herminghaus, Topological microfluidics for flexible micro-cargo concepts, Soft Matter 9, 7251 (2013).

[21] F. Woodhouse and J. Dunkel, Active matter logic for autonomous microfluidics, Nat. Commun. 8, 15169 (2017).

[22] A. Doostmohammadi, T. N. Shendruk, K. Thijssen, and J. M. Yeomans, Onset of meso-scale turbulence in active nematics, Nat. Commun. 8, 15326 (2017).

[23] S. Chandragiri, A. Doostmohammadi, J. M. Yeomans, and S. P. Thampi, Flow States and Transitions of an Active Nematic in a Three-Dimensional Channel, Phys. Rev. Lett. 125, 148002 (2020).

[24] N. J. Mottram and C. J. P. Newton, Introduction to q-tensor theory, arXiv:1409.3542.

[25] D. Vincenzi, P. Perlekar, L. Biferale, and F. Toschi, Impact of the Peterlin approximation on polymer dynamics in turbulent flows, Phys. Rev. E 92, 053004 (2015).

[26] S. Succi, The Lattice Boltzmann Equation: For Fluid Dynamics and Beyond (Oxford University Press, Oxford, 2001).

[27] L. Giomi, Geometry and topology of turbulence in active nematics, Phys. Rev. X 5, 031003 (2015). 
[28] R. Alert, J.-F. Joanny, and J. Casademunt, Universal scaling of active nematic turbulence, Nat. Phys. 16, $682(2020)$.

[29] T. N. Shendruk, A. Doostmohammadi, K. Thijssen, and J. M. Yeomans, Dancing disclinations in confined active nematics, Soft Matter 13, 3853 (2017).

[30] S. P. Thampi, R. Golestanian, and J. M. Yeomans, Driven active and passive nematics, Mol. Phys. 113, 2656 (2015).

[31] S. Ramaswamy and M. Rao, Active-filament hydrodynamics: Instabilities, boundary conditions and rheology, New J. Phys. 9, 423 (2007).

[32] J. V. Selinger, Introduction to the Theory of Soft Matter From Ideal Gases to Liquid Crystals (Springer, 2015).

[33] S. Chandragiri, A. Doostmohammadi, J. M. Yeomans, and S. P. Thampi, Active transport in a channel: Stabilisation by flow or thermodynamics, Soft Matter 15, 1597 (2019). 